\title{
Collective Tree Spanners and Routing in AT-free Related Graphs
}

\author{
Feodor F. Dragan ${ }^{1}$, Chenyu Yan ${ }^{1}$, and Derek G. Corneil ${ }^{2}$ \\ 1 Department of Computer Science, Kent State University, Kent, Ohio, USA \\ \{dragan, cyan\}@cs. kent. edu \\ 2 Department of Computer Science, University of Toronto, Toronto, Ontario, Canada \\ dgc@cs.toronto.edu
}

\begin{abstract}
In this paper we study collective additive tree spanners for families of graphs that either contain or are contained in AT-free graphs. We say that a graph $G=(V, E)$ admits a system of $\mu$ collective additive tree $r$-spanners if there is a system $\mathcal{T}(G)$ of at most $\mu$ spanning trees of $G$ such that for any two vertices $x, y$ of $G$ a spanning tree $T \in \mathcal{T}(G)$ exists such that $d_{T}(x, y) \leq d_{G}(x, y)+r$. Among other results, we show that AT-free graphs have a system of two collective additive tree 2-spanners (whereas there are trapezoid graphs that do not admit any additive tree 2-spanner). Furthermore, based on this collection of trees, we derive a compact and efficient routing scheme for those graphs. Also, any DSPgraph (there exists a dominating shortest path) admits one additive tree 4 -spanner, a system of two collective additive tree 3 -spanners and a system of five collective additive tree 2 -spanners.
\end{abstract}

\section{Introduction}

Given a graph $G=(V, E)$, a spanning subgraph $H$ is called a spanner if $H$ provides a "good" approximation of the distances in $G$. More formally, for $t \geq 1$, $H$ is called a multiplicative t-spanner of $G[1,14,13]$ if $d_{H}(u, v) \leq t \cdot d_{G}(u, v)$ for all $u, v \in V$. If $r \geq 0$ and $d_{H}(u, v) \leq d_{G}(u, v)+r$ for all $u, v \in V$, then $H$ is called an additive $r$-spanner of $G$ [8]. The parameters $t$ and $r$ are called, respectively, the multiplicative and the additive stretch factors. Clearly, every additive $r$-spanner of $G$ is a multiplicative $(r+1)$-spanner of $G$ (but not vice versa). In this paper, we continue the approach taken in [4] of studying collective tree spanners. We say that a graph $G=(V, E)$ admits a system of $\mu$ collective additive tree $r$-spanners if there is a system $\mathcal{T}(G)$ of at most $\mu$ spanning trees of $G$ such that for any two vertices $x, y$ of $G$ a spanning tree $T \in \mathcal{T}(G)$ exists such that $d_{T}(x, y) \leq d_{G}(x, y)+r$ (a multiplicative variant of this notion can be defined analogously). Clearly, if $G$ admits a system of $\mu$ collective additive tree $r$-spanners, then $G$ admits an additive $r$-spanner with at most $\mu \times(n-1)$ edges (take the union of all those trees), and if $\mu=1$ then $G$ admits an additive tree $r$ spanner. Note also that any graph on $n$ vertices admits a system of at most $n-1$ collective additive tree 0 -spanners (take $n-1$ Breadth-First-Search-trees rooted at different vertices of $G$ ). In particular, we examine the problem of finding small 
systems of collective additive tree $r$-spanners for small values of $r$ on classes of graphs that are related to the well known asteroidal triple-free (AT-free) graphs, notably the restricted families: permutation graph and trapezoid graphs and the generalizations: DSP-graphs and graphs with bounded asteroidal number.

Once one has determined a system of collective additive tree spanners, it is interesting to see how such a system can be used to design compact and efficient routing schemes for the given graph. Following [12], one can give the following formal definition. A family $\Re$ of graphs is said to have an $l(n)$-bit routing labeling scheme if there is a function $L$ labeling the vertices of each $n$-vertex graph in $\Re$ with distinct labels of up to $l(n)$ bits, and there exists an efficient algorithm, called the routing decision, that given the label of a source vertex $v$ and the label of the destination vertex (the header of the packet), decides in time polynomial in the length of the given labels and using only those two labels, whether this packet has already reached its destination, and if not, to which neighbor of $v$ to forward the packet. The quality of a routing scheme is measured in terms of its additive stretch, called deviation, (or multiplicative stretch, called delay), namely, the maximum surplus (or ratio) between the length of a route, produced by the scheme for some pair of vertices, and their distance.

\subsection{Our Results}

After introducing the notation and definitions used throughout the paper, we examine various families of graphs related to AT-free graphs from the perspective of determining whether they have a small constant number of collective additive tree $r$-spanners for small constant $r$. In Section 2 we show that AT-free graphs have a system of two collective additive tree 2 -spanners, permutation graphs have a single additive tree 2 -spanner but there are trapezoid graphs that do not admit any additive tree 2-spanner (thereby disproving a conjecture of [15]). All of these tree spanners can be easily constructed in linear time. For families that strictly contain AT-free graphs, we prove that any DSP-graph admits one additive tree 4 -spanner, a system of two collective additive tree 3 -spanners and a system of five collectible additive tree 2 -spanners. Furthermore, any graph $G$ with asteroidal number an $(G)$ admits a system of an $(G)(\operatorname{an}(G)-1) / 2$ collective additive tree 4-spanners and a system of an $(G)(\operatorname{an}(G)-1)$ collective additive tree 3-spanners. In Section 3, we show how the system of two collective additive tree 2-spanners for AT-free graphs can be used to derive a compact and efficient routing scheme. In particular we will show that any AT-free graph with diameter $\mathcal{D}$ and maximum vertex degree $\Delta$ admits a $\left(3 \log _{2} \mathcal{D}+6 \log _{2} \Delta+O(1)\right)$-bit routing labeling scheme of deviation at most 2. Moreover, the scheme is computable in linear time, and the routing decision is made in constant time per vertex.

\subsection{Basic Notions and Notation}

All graphs occurring in this paper are connected, finite, undirected, loopless and without multiple edges. In a graph $G=(V, E)$ the length of a path from a vertex $v$ to a vertex $u$ is the number of edges in the path. The distance $d_{G}(u, v)$ between the vertices $u$ and $v$ is the length of a shortest path connecting $u$ and 
$v$. The eccentricity $\operatorname{ecc}(v)$ of a vertex $v$ of $G$ is $\max _{u \in V} d_{G}(u, v)$. The diameter $\operatorname{diam}(G)$ of $G$ is $\max _{v \in V} \operatorname{ecc}(v)$. The $i$ th neighborhood of a vertex $v$ of $G$ is the set $N_{i}(v):=\left\{u \in V: d_{G}(v, u)=i\right\}$. For a vertex $v$ of $G$, the sets $N(v):=N_{1}(v)$ and $N[v]:=N(v) \cup\{v\}$ are called the open neighborhood and the closed neighborhood of $v$, respectively. For a set $S \subseteq V$, by $N[S]:=\bigcup_{v \in S} N[v]$ we denote the closed neighborhood of $S$ and by $N(S):=N[S] \backslash S$ the open neighborhood of $S$. A set $D \subseteq V$ is called a dominating set of a graph $G=(V, E)$ if $N[D]=V$.

An independent set of three vertices such that each pair is joined by a path that avoids the neighborhood of the third is called an asteroidal triple. A graph $G$ is an AT-free graph if it does not contain any asteroidal triples [2]. In [7], the notion of asteroidal triple was generalized. An independent set $A \subseteq V$ of a graph $G=(V, E)$ is called an asteroidal set of $G$ if for each $a \in A$ the vertices of $A \backslash\{a\}$ are contained in one connected component of $G-N[a]$, the graph obtained from $G$ by removing vertices of $N[a]$. The maximum cardinality of an asteroidal set of $G$ is denoted by an $(G)$, and called the asteroidal number of $G$. The class of graphs of bounded asteroidal number extends naturally the class of AT-free graphs; AT-free graphs are exactly the graphs with asteroidal number at most two.

Let $P$ be a shortest path of $G$. If every vertex $z$ of $G$ belongs to the neighborhood $N[P]$ of $P$, then we say that $P$ is a dominating shortest path of $G$. A graph $G$ is called a Dominating-Shortest-Path-graph (or DSP-graph, for short), if it has a dominating shortest path. By the Dominating Pair Theorem given in [2], any AT-free graph is a DSP-graph.

The class of AT-free graphs contains many intersection families of graphs, among them the permutation graphs, the trapezoid graphs and the cocomparability graphs. These three families of graphs can be defined as follows. Consider two parallel lines (upper and lower) in the plane. Assume that each line contains $n$ points, labeled 1 to $n$, and each two points with the same label define a segment with that label. The intersection graph of such a set of segments between two parallel lines is called a permutation graph. Assume now that each line contains $n$ intervals, labeled 1 to $n$, and each two intervals with the same label define a trapezoid with that label (a trapezoid can degenerate to a triangle or to a segment). The intersection graph of such a set of trapezoids between two parallel lines is called a trapezoid graph. Clearly, every permutation graph is a trapezoid graph, but not vice versa. The class of cocomparability graphs (which contains all trapezoid graphs as a subclass) can be defined as the intersection graphs of continuous function diagrams, but for this paper it would be more convenient to define them via the existence of a special vertex ordering. A graph $G$ is a cocomparability graph if it admits a vertex ordering $\sigma=\left[v_{1}, v_{2}, \ldots, v_{n}\right]$, called a cocomparability ordering, such that for any $i<j<k$, if $v_{i}$ is adjacent to $v_{k}$ then $v_{j}$ must be adjacent to $v_{i}$ or to $v_{k}$. According to [11], such an ordering of a cocomparability graph can be constructed in linear time. Note also that, given a permutation graph $G$, a permutation model (i.e., a set of segments between two parallel lines, defining $G$ ) can be found in linear time [11]. A trapezoid model for a trapezoid graph can be found in $O\left(n^{2}\right)$ time [9]. 


\section{Collective Additive Tree Spanners}

\subsection{AT-free Graphs}

It is known [15] that any AT-free graph admits one additive tree 3-spanner. In this subsection we show that any AT-free graph admits a system of two collective additive tree 2-spanners.

As a consequence of the Dominating Pair Theorem given in [2], any AT-free graph has a dominating shortest path which can be found in linear time by $2 \times$ LexBFS [3]. The $2 \times$ LexBFS method first starts a lexicographic breadthfirst search (LexBFS) from an arbitrary vertex $x$ of $G$ and then starts a second LexBFS from the vertex $x_{0}$ last visited by the first LexBFS. Let $x_{l}$ be the vertex of $G$ last visited by the second LexBFS. As shown in [3], every shortest path $\left(x_{0}, x_{1}, \ldots, x_{l}\right)$, connecting $x_{0}$ and $x_{l}$, is a dominating shortest path of $G$. Next we demonstrate how to use such a dominating shortest path in an AT-free graph to show that every AT-free graph admits a system of two collective additive tree 2 -spanners. We will need the following result from [6].

Lemma 1. [6] Let $P:=\left(x_{0}, x_{1}, \ldots, x_{l}\right)$ be a dominating shortest path of an ATfree graph $G=(V, E)$ constructed by $2 \times \operatorname{LexBFS}$. Then, for every $i=1,2, \ldots, l$, every vertex $z \in N_{i}\left(x_{0}\right)$ is adjacent to $x_{i}$ or $x_{i-1}$.

Using this lemma, we construct a first spanning tree $T_{1}=\left(V, E_{1}\right)$ for an AT-free graph $G=(V, E)$ as follows: put into initially empty $E_{1}$ all edges of the path $P:=\left(x_{0}, x_{1}, \ldots, x_{l}\right)$, and then for each vertex $z \in N_{i}\left(x_{0}\right)$, put edge $z x_{i-1}$ into $E_{1}$, if $z$ is adjacent to $x_{i-1}$ in $G$, and put edge $z x_{i}$ into $E_{1}$, otherwise. We call this spanning tree the caterpillar-tree of $G$ (with spine $P$ ). According to [15], this caterpillar-tree gives already an additive tree 3 -spanner for the ATfree graph $G$. To get a collective additive stretch factor 2 for $G$, we construct a second spanning tree $T_{2}=\left(V, E_{2}\right)$ for $G$ as follows. Set $L_{i}:=N_{i}\left(x_{0}\right)$ for each $i=1,2, \ldots, l$.

set $E_{2}:=\left\{\right.$ all edges of the path $\left.P:=\left(x_{0}, x_{1}, \ldots, x_{l}\right)\right\}$;

set $\operatorname{dev}\left(x_{i}\right):=0$ for each vertex $x_{i}$ of the path $P$;

for $i=1$ to $l$ do

for each vertex $z \in L_{i} \backslash\left\{x_{i}\right\}$ do

among all neighbors of $z$ in $L_{i-1}$ choose a neighbor $w$ with minimum deviation $\operatorname{dev}(w)$;

add edge $z w$ to $E_{2}$ and set $\operatorname{dev}(z):=\operatorname{dev}(w)+1$;

enddo

enddo.

We call spanning tree $T_{2}$ the cactus-tree of $G$ (with stem $P$ ). It is evident, by construction, that the cactus-tree $T_{2}$ is a special kind of breadth-first-search-tree of $G$. The value $\operatorname{dev}(z)$ (called the deviation of $z$ from stem $P$ ) gives the distance in $T_{2}$ between vertex $z$ and path $P$. In Figure 1 we show an AT-free graph $G$ along with its caterpillar-tree $T_{1}$ and cactus-tree $T_{2}$.

Lemma 2. Spanning trees $\left\{T_{1}, T_{2}\right\}$ are collective additive tree 2-spanners of AT-free graph $G$. 


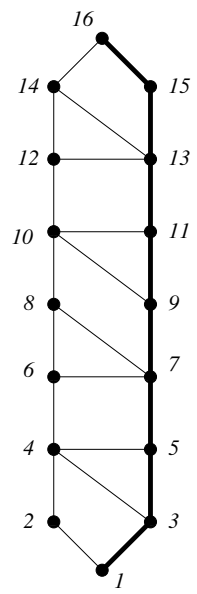

(a)

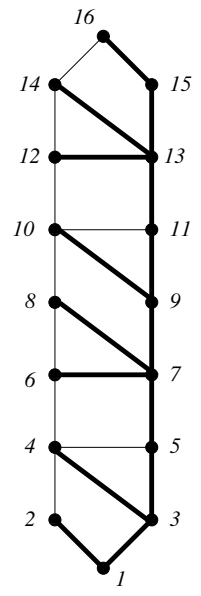

(b)

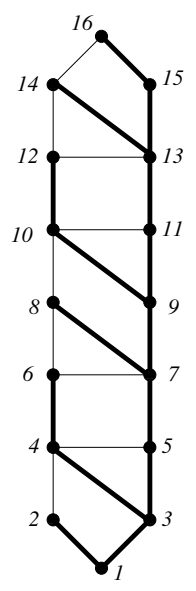

(c)

Fig. 1. (a) An AT-free graph $G$ with a dominating path $P$, (b) the caterpillar-tree $T_{1}$ of $G$ and (c) the cactus-tree $T_{2}$ of $G$

Proof. Consider two arbitrary vertices $x \in L_{i}$ and $y \in L_{j}(y \neq x)$ of $G$, where $j \leq i$. If $i=j$, i.e., both $x$ and $y$ lie in the same layer $L_{i}=L_{j}$, then the distance in $T_{1}$ between $x$ and $y$ is at most 3 , since in the worst case one of them is adjacent to $x_{i}$ in $T_{1}$ and the second to $x_{i-1}$. Thus, $d_{T_{1}}(x, y) \leq 3 \leq d_{G}(x, y)+2$ holds when $i=j$, and therefore, we may assume that $i>j$.

We know that $d_{G}(x, y) \geq i-j$. By the construction of the caterpillar-tree $T_{1}$, we have $d_{T_{1}}\left(y, x_{j}\right) \leq 2$ and $d_{T_{1}}\left(x, x_{i-1}\right) \leq 2$. Hence, $d_{T_{1}}(x, y) \leq d_{T_{1}}\left(x, x_{i-1}\right)+$ $d_{T_{1}}\left(x_{i-1}, x_{j}\right)+d_{T_{1}}\left(y, x_{j}\right) \leq 2+i-1-j+2 \leq d_{G}(x, y)+3$, and equality $d_{T_{1}}(x, y)=d_{G}(x, y)+3$ holds if and only if $d_{G}(x, y)=i-j$, vertex $x$ is adjacent to $x_{i}$ in $T_{1}$ (and thus in $G$, vertex $x$ is not adjacent to $x_{i-1}$ ) and vertex $y$ is adjacent to $x_{j-1}$ in $T_{1}$ but does not coincide with $x_{j}$. We will show that in this case in the cactus-tree $T_{2}, d_{T_{2}}(x, y) \leq d_{G}(x, y)+2$.

Consider in $G$ a shortest path $\left(y=y_{0}, y_{1}, \ldots, y_{i-j}=x\right)$ connecting vertices $y$ and $x$. Clearly, $y_{k} \in L_{j+k}$ for each $k=0,1, \ldots, i-j-1$, and since $y_{k}$ is a neighbor of $y_{k+1}$ in layer $L_{j+k}$, by construction of $T_{2}$, we have $\operatorname{dev}\left(y_{0}\right)=1$ and $\operatorname{dev}\left(y_{k+1}\right) \leq \operatorname{dev}\left(y_{k}\right)+1 \leq k+2$. Hence, the deviation of vertex $x$ is at most $i-j+1$. That is, there is a path in $T_{2}$ between $x$ and a stem vertex $x_{s}$ $(j-1 \leq s \leq i-2)$ of length $i-s$. The latter implies the existence in $T_{2}$ of a path of length $i-j+1$ between vertices $x$ and $x_{j-1}$. Therefore, $d_{T_{2}}(x, y) \leq$ $d_{T_{2}}\left(x, x_{j-1}\right)+1=i-j+1+1=d_{G}(x, y)+2$.

From this lemma we immediately conclude.

Theorem 1. Any AT-free graph admits a system of two collective additive tree 2-spanners, constructable in linear time.

In the next subsection, we will show that to get a collective additive stretch factor 2 for some AT-free graphs, one needs at least two spanning trees. There- 
fore, the result given in Theorem 1 is best possible. Furthermore, to achieve a collective additive stretch factor 1 or 0 for some AT-free graphs, one needs $\Omega(n)$ spanning trees.

\subsection{Permutation Graphs and Trapezoid Graphs}

It is known [10] that any permutation graph admits a multiplicative tree 3spanner. In this subsection, we show that any permutation graph admits an additive tree 2-spanner and any system of collective additive tree 1-spanners must have $\Omega(n)$ spanning trees for some permutation graphs. Here also we disprove a conjecture given in [15], that any cocomparability graph admits an additive tree 2 -spanner. We show that there exists even a trapezoid graph which does not admit any additive tree 2-spanner.

Let $G=(V, E)$ be a permutation graph given together with a permutation model. In what follows, "u.p." and "l.p." refer to a vertex's point on the upper and lower, respectively, line of the permutation model. Construct BFS-layers $\left(\left\{L_{0}, L_{1}, \cdots\right\}\right)$ and the spine $\left\{x_{1}, x_{2}, \cdots\right\}$ of $G$ as follows (the process continues until $\left.L_{i}=\emptyset\right)$.

set $x_{0}:=$ the vertex whose u.p. is as far left as possible;

set $L_{0}:=\left\{x_{0}\right\}$;

set $L_{1}:=\left\{\right.$ vertices whose l.p.s are to the left of the l.p. of $\left.x_{0}\right\}$;

set $x_{1}:=$ the vertex in $L_{1}$ with the u.p. as far right as possible;

set $L_{2}:=\left\{\right.$ vertices whose u.p.s are between the u.p.s of $x_{0}$ and $\left.x_{1}\right\} \backslash L_{1}$;

set $x_{2}:=$ the vertex in $L_{2}$ with the l.p. as far right as possible;

for $i=3$ to $n$ do

if $i$ is odd then

set $L_{i}:=\left\{\right.$ vertices with l.p. between the l.p.s of $x_{i-3}$ and $\left.x_{i-1}\right\} \backslash L_{i-1}$;

set $x_{i}:=$ the vertex in $L_{i}$ with the u.p. as far right as possible; else

set $L_{i}:=\left\{\right.$ vertices whose u.p.s are between the u.p.s of $x_{i-3}$ and $\left.x_{i-1}\right\} \backslash L_{i-1}$;

set $x_{i}:=$ the vertex in $L_{i}$ with the l.p. as far right as possible; enddo.

It is straightforward to show that for all $i \geq 0$, if $y \in L_{i+1}$ then $x_{i} y \in E$. Now by forming $T$ to include all edges from $x_{i}$ to $L_{i+1}$ for appropriate $i$, we can conclude: (The details will be in the journal version of the paper.)

Theorem 2. Every permutation graph admits an additive tree 2-spanner, constructable in linear time.

In the journal version we show that there exists a trapezoid graph which does not admit any additive tree 2-spanner, thereby disproving a conjecture from [15] that any cocomparability graph admits an additive tree 2 -spanner. We show also that there are bipartite permutation graphs on $2 n$ vertices for which any system of collective additive tree 1 -spanners will need to have at least $\Omega(n)$ spanning trees. 


\subsection{DSP-graphs}

It follows from a result in [15] that any DSP-graph admits one additive tree 4 -spanner. In this subsection we show that any DSP-graph admits a system of two collective additive tree 3 -spanners and a system of five collective additive tree 2-spanners.

Let $G=(V, E)$ be a DSP-graph and $P:=\left(v=x_{0}, x_{1}, \ldots, x_{l}=u\right)$ be a dominating shortest path of $G$. We will build five spanning trees $\left\{T_{1}, T_{2}, T_{3}, T_{4}, T_{5}\right\}$ for $G$, all containing the edges of $P$, in such a way that for any two vertices $x, y \in V$, there will be a tree $T^{\prime} \in\left\{T_{1}, T_{2}, T_{3}, T_{4}, T_{5}\right\}$ with $d_{T^{\prime}}(x, y) \leq d_{G}(x, y)+2$.

Our first three trees $T_{1}, T_{2}, T_{3}$ are very similar to the trees constructed for AT-free graphs. The tree $T_{1}=\left(V, E_{1}\right)$ is constructed as follows. Add to initially empty set $E_{1}$ all edges of path $P$. Then, for each vertex $z \in V \backslash P$ choose an arbitrary neighbor $w_{z}$ in $P$ and add edge $z w_{z}$ to $E_{1}$. The tree $T_{1}$ is an analog of the caterpillar-tree constructed for an AT-free graph. The second and third trees are analogs of the cactus-tree considered for an AT-free graph. The tree $T_{2}=\left(V, E_{2}\right)$ is a special breadth-first-search-tree $T_{v}$ with vertex $v$ as the root, the tree $T_{3}=\left(V, E_{3}\right)$ is a special breadth-first-search-tree $T_{u}$ with vertex $u$ as the root. For construction of $T_{2}$ we can use the algorithm given in Subsection 2.1 with one additional line at the end: for each $z \in N_{l+1}(v)$, add edge $z u$ to $E_{2}$ and set $\operatorname{dev}(z):=1 . T_{3}$ is constructed similarly; we simply reverse the order of vertices of $P$ and consider $u$ instead of $v$ and $E_{3}$ instead of $E_{2}$. The detailed algorithm will appear in the journal version.

Our tree $T_{4}=\left(V, E_{4}\right)$ is a generalization of the tree $T_{2}$ and is constructed as follows.

set $E_{4}:=\left\{\right.$ all edges of the path $\left.P:=\left(v=x_{0}, x_{1}, \ldots, x_{l}=u\right)\right\}$;

set $\operatorname{dev}\left(x_{i}\right):=0$ for each vertex $x_{i}$ of the path $P$;

for $i=1$ to $l$ do

for each vertex $z \in N_{i}(v) \backslash\left\{x_{i}\right\}$ do case

case $\left(z\right.$ is adjacent to $x_{i-1}$ in $G$ )

add edge $z x_{i-1}$ to $E_{4}$ and set $\operatorname{dev}(z):=1$;

case $\left(z\right.$ is adjacent to $x_{i}$ in $G$ )

add edge $z x_{i}$ to $E_{4}$ and set $\operatorname{dev}(z):=1$;

case ( $z$ is adjacent to a vertex $w \in N_{i}(v)$ which is adjacent to $\left.x_{i-1}\right)$

choose such a $w$ and add edge $z w$ to $E_{4}$ and set $\operatorname{dev}(z):=2$;

otherwise /* none of above */

among all neighbors of $z$ in $N_{i-1}(v)$ choose a neighbor $w$ with

minimum deviation $\operatorname{dev}(w)$ (break ties arbitrarily);

add edge $z w$ to $E_{4}$ and set $\operatorname{dev}(z):=\operatorname{dev}(w)+1$;

endcase

enddo

for each $z \in N_{l+1}(v)$, add edge $z u$ to $E_{4}$ and set $\operatorname{dev}(z):=1$.

It is an easy exercise to show by induction that for any vertex $z \in N_{i}(v)$, the vertex of $P$ closest to $z$ in $T_{4}$ is either $x_{s}$ or $x_{s-1}$ with $s=i-\operatorname{dev}(z)+1$. Moreover, the length of the path of $T_{4}$ between $z$ and $P$ is $\operatorname{dev}(z)$. Our last tree $T_{5}=\left(V, E_{5}\right)$ is a version of the tree $T_{4}$, constructed downwards. 
set $E_{5}:=\left\{\right.$ all edges of the path $\left.P:=\left(v=x_{0}, x_{1}, \ldots, x_{l}=u\right)\right\}$;

set $\operatorname{dev}\left(x_{i}\right):=0$ for each vertex $x_{i}$ of the path $P$ and $\operatorname{dev}(z):=\infty$ for any $z \in V \backslash P$; for $i=l-1$ down to 1 do

for each vertex $z \in N_{i}(v) \backslash\left\{x_{i}\right\}$ do case

case ( $z$ is adjacent to $x_{i+1}$ in $G$ )

add edge $z x_{i+1}$ to $E_{5}$ and set $\operatorname{dev}(z):=1$;

case ( $z$ is adjacent to $x_{i}$ in $G$ )

add edge $z x_{i}$ to $E_{5}$ and set $\operatorname{dev}(z):=1$;

case ( $z$ is adjacent to a vertex $w \in N_{i}(v)$ which is adjacent to $x_{i+1}$ )

choose such a $w$ and add edge $z w$ to $E_{5}$ and set $\operatorname{dev}(z):=2$;

otherwise

if $z$ has neighbors in $N_{i+1}(v)$ then

among all neighbors of $z$ in $N_{i+1}(v)$ choose a neighbor $w$ with minimum deviation $\operatorname{dev}(w)$ (break ties arbitrarily);

if $\operatorname{dev}(w)<\infty$ then add edge $z w$ to $E_{5}$ and set $\operatorname{dev}(z):=\operatorname{dev}(w)+1$;

endcase

enddo

for each vertex $z$ with $\operatorname{dev}(z)$ still $\infty$ do

let $z \in N_{i}(v)$;

if $i=l$ and $z$ is adjacent to $x_{l}$ then add edge $z x_{l}$ to $E_{5}$ and set $\operatorname{dev}(z):=1$;

else add edge $z x_{i-1}$ to $E_{5}$;

${ }^{*}$ this edge exists in $G$ since $P$ is a dominating path

and $z$ is adjacent in $G$ neither to $x_{i+1}$ nor $x_{i} * /$

enddo.

Again, it is easy to see that for any vertex $z \in N_{i}(v)$ with finite deviation $\operatorname{dev}(z)$, the vertex of $P$ closest to $z$ in $T_{5}$ is either $x_{s}$ or $x_{s+1}$ with $s=i+\operatorname{dev}(z)-1$. The length of the path of $T_{5}$ between $z$ and $P$ is $\operatorname{dev}(z)$.

We are ready to present the main result of this subsection.

Lemma 3. Let $G$ be a DSP-graph with a dominating shortest path $P:=(v=$ $\left.x_{0}, x_{1}, \ldots, x_{l}=u\right)$ and spanning trees $T_{1}, T_{2}, T_{3}, T_{4}, T_{5}$ constructed starting from $P$ as described above. Then, for any two vertices $x, y \in V$ :

1. $d_{T_{1}}(x, y) \leq d_{G}(x, y)+4$;

2. there is a tree $T^{\prime} \in\left\{T_{1}, T_{2}\right\}$ such that $d_{T^{\prime}}(x, y) \leq d_{G}(x, y)+3$;

3. there is a tree $T^{\prime \prime} \in\left\{T_{1}, T_{2}, T_{3}, T_{4}, T_{5}\right\}$ such that $d_{T^{\prime \prime}}(x, y) \leq d_{G}(x, y)+2$.

Proof. The proof of this lemma is quite technical and will appear in the journal version of the paper.

Theorem 3. Any DSP-graph admits one additive tree 4-spanner, a system of two collective additive tree 3-spanners and a system of five collective additive tree 2-spanners. Moreover, given a dominating shortest path of $G$, all trees are constructable in linear time.

Note that there is a DSP-graph for which two trees are necessary to get a collective additive stretch factor 3 . However, it is an open question whether to achieve a collective additive stretch factor 2 , one really needs five spanning trees. 


\subsection{Graphs with Bounded Asteroidal Number}

It is known [7] that any graph $G$ with asteroidal number an $(G)$ admits an additive tree $(\operatorname{3an}(G)-1)$-spanner. In this subsection we show that any graph with asteroidal number an $(G)$ admits a system of an $(G)(\operatorname{an}(G)-1) / 2$ collective additive tree 4 -spanners and a system of an $(G)(\operatorname{an}(G)-1)$ collective additive tree 3-spanners.

In what follows we will use the following definitions and results from [7]. An asteroidal set $A$ of a graph $G$ is repulsive if for every vertex $v \in V \backslash N[A]$, not all vertices of $A$ are contained in one connected component of $G-N[v]$. According to [7], any graph has a repulsive asteroidal set. A set $D \subseteq V$ in a graph $G$ is said to be a dominating target, if $D \cup S$ is a dominating set in $G$ for every set $S \subseteq V$ for which the subgraph of $G$ induced by $D \cup S$ is connected. It is shown in [7] that any graph $G$ has a dominating target $D$ with $D \leq \operatorname{an}(G)$. Furthermore, every repulsive asteroidal set of $G$ is such a dominating target of $G$.

In the journal version of the paper, we prove the following stronger version of the result above. Let $D \subseteq V$ be a repulsive asteroidal set of a graph $G=(V, E)$.

Lemma 4. For every $x, y \in V$, there exist $a, b \in D$ such that $x, y \in N[P]$ for any path $P$ of $G$ between $a$ and $b$.

Consider two arbitrary vertices $a, b$ of $D$ and a shortest path $P(a, b):=(a=$ $\left.x_{0}, x_{1}, \ldots, x_{l}=b\right)$ connecting $a$ and $b$ in $G$. We can build two spanning trees $T_{1}(a, b)$ and $T_{2}(a, b)$ for $G$, both containing the edges of $P(a, b)$, in such a way that for any two vertices $x, y \in N[P(a, b)], d_{T_{1}(a, b)}(x, y) \leq d_{G}(x, y)+4$ and $\min \left\{d_{T_{1}(a, b)}(x, y), d_{T_{2}(a, b)}(x, y)\right\} \leq d_{G}(x, y)+3$.

Our trees $T_{1}(a, b)$ and $T_{2}(a, b)$ are very similar to the trees constructed for ATfree graphs. The tree $T_{1}(a, b)=\left(V, E_{1}\right)$ is constructed as follows. Add to initially empty set $E_{1}$ all edges of path $P(a, b)$. Then, for each vertex $z \in N(P(a, b))$ choose an arbitrary neighbor $w$ in $P(a, b)$ and add edge $z w$ to $E_{1}$. The obtained subtree of $G$ (which covers so far only vertices from $N[P(a, b)]$ ) extends to a spanning tree $T_{1}(a, b)$ arbitrarily. The tree $T_{1}(a, b)$ is an analog of the caterpillartree constructed for an AT-free graph. The second tree is an analog of the cactustree considered for an AT-free graph. The tree $T_{2}(a, b)=\left(V, E_{2}\right)$ is a special breadth-first-search-tree $T_{a}$ with vertex $a$ as the root. The detailed algorithm for constructing $T_{2}(a, b)$ and the proof of the following lemma will appear in the journal version.

Lemma 5. For any two vertices $x, y \in N[P(a, b)]$ :

1. $d_{T_{1}(a, b)}(x, y) \leq d_{G}(x, y)+4$;

2. there is a tree $T^{\prime} \in\left\{T_{1}(a, b), T_{2}(a, b)\right\}$ such that $d_{T^{\prime}}(x, y) \leq d_{G}(x, y)+3$.

If we construct trees $T_{1}(a, b)$ and $T_{2}(a, b)$ for each pair of vertices $a, b \in D$, from Lemma 4 and Lemma 5 , we get (recall that $|D| \leq$ an $(G)$ ):

Theorem 4. Any graph $G$ with asteroidal number an $(G)$ admits a system of $\operatorname{an}(G)(\operatorname{an}(G)-1) / 2$ collective additive tree 4-spanners and a system of $\operatorname{an}(G)(\operatorname{an}(G)-1)$ collective additive tree 3-spanners. 
Corollary 1. Any graph $G$ with asteroidal number bounded by a constant admits a system of a constant number of collective additive tree 3-spanners. Moreover, given a repulsive asteroidal set of $G$, all trees are constructable in total linear time.

\section{Routing Labeling Schemes in AT-free Graphs}

In this section, we use the results obtained above to design compact and efficient routing labeling schemes for AT-free graphs. For DSP-graphs and graphs with bounded asteroidal number, corresponding routing labeling schemes are described in the journal version of the paper. We will show that any AT-free graph with diameter $\mathcal{D}:=\operatorname{diam}(G)$ and maximum vertex degree $\Delta$ admits a $\left(3 \log _{2} \mathcal{D}+6 \log _{2} \Delta+O(1)\right)$-bit routing labeling scheme of deviation at most 2 . Moreover, the scheme is computable in linear time, and the routing decision is made in constant time per vertex.

It is worth mentioning that any AT-free graph admits a $\left(\log _{2} \mathcal{D}+1\right)$-bit distance labeling scheme of deviation at most 2 (see [5]). That is, there is a function $L$ labeling the vertices of each AT-free graph $G$ with (not necessarily distinct) labels of up to $\log _{2} \mathcal{D}+1$ bits such that given two labels $L(v), L(u)$ of two vertices $v, u$ of $G$, it is possible to compute in constant time, by merely inspecting the labels of $u$ and $v$, a value $\widehat{d}(u, v)$ such that $0 \leq \widehat{d}(u, v)-d_{G}(u, v) \leq 2$. To the best of our knowledge, the method of [5] cannot be used (at least directly) to design a routing labeling scheme for AT-free graphs.

Labels. In subsection 2.1, we showed that any AT-free graph $G=(V, E)$ admits a system of two collective additive tree 2-spanners. During the construction of the cactus-tree $T_{2}$ for $G$, each vertex $z \in V$ received a deviation number $\operatorname{dev}(z)$ which is the distance in $T_{2}$ between $z$ and the stem $P:=\left(x_{0}, x_{1}, \ldots, x_{l}\right)$ of $T_{2}$. To simplify the routing decision, it will be useful to construct one more spanning tree $T^{\prime}=\left(V, E^{\prime}\right)$ for $G$. Let $P:=\left(x_{0}, x_{1}, \ldots, x_{l}\right)$ be the dominating path of $G$ described in Lemma 1.

set $E^{\prime}:=\left\{\right.$ all edges of the path $\left.P:=\left(x_{0}, x_{1}, \ldots, x_{l}\right)\right\}$;

set $\operatorname{dev}^{\prime}\left(x_{i}\right):=0$ for each $x_{i}$ of the path $P$ and $\operatorname{dev}^{\prime}(z):=l+1$ for any $z \in V \backslash P$;

for each $z \in N_{l}\left(x_{0}\right)$ which is adjacent to $x_{l}$, set $\operatorname{dev}^{\prime}(z):=1$ and add edge $z x_{l}$ to $E^{\prime}$; for $i=l-1$ down to 1 do

for each vertex $z \in N_{i}\left(x_{0}\right) \backslash\left\{x_{i}\right\}$ do

if $z$ is adjacent to $x_{i}$ in $G$ then add edge $z x_{i}$ to $E^{\prime}$ and set $\operatorname{dev}^{\prime}(z):=1$;

else if $z$ has neighbors in $N_{i+1}\left(x_{0}\right)$ then

among all neighbors of $z$ in $N_{i+1}\left(x_{0}\right)$, choose a neighbor $w$ with minimum deviation $\operatorname{dev}^{\prime}(w)$ (break ties arbitrarily);

if $\operatorname{dev}^{\prime}(w)<l+1$ then add edge $z w$ to $E^{\prime}$ and set $\operatorname{dev}^{\prime}(z):=\operatorname{dev}^{\prime}(w)+1$;

enddo

enddo

for each vertex $z$ with $\operatorname{dev}^{\prime}(z)$ still $l+1$ do

let $z \in N_{i}\left(x_{0}\right)$;

add edge $z x_{i-1}$ to $E^{\prime}$;

enddo. 
We name tree $T^{\prime}$ the willow-tree of $G$. As a result of its construction, each vertex $z \in V$ received a second deviation number $\operatorname{dev}^{\prime}(z)$, which is either $l+1$ or the distance in $T^{\prime}$ between $z$ and the path $P:=\left(x_{0}, x_{1}, \ldots, x_{l}\right)$ of $T^{\prime}$.

Now we are ready to describe the routing labels of the vertices of $G$. For each vertex $x_{i} \in P(i=0,1, \ldots, l)$, we have

$$
\operatorname{Label}\left(x_{i}\right):=\left(b\left(x_{i}\right), \operatorname{level}\left(x_{i}\right), \operatorname{port}_{u p}\left(x_{i}\right), \operatorname{port}_{\text {down }}\left(x_{i}\right)\right),
$$

where

$-b\left(x_{i}\right):=1$, a bit indicating that $x_{i}$ belongs to $P$;

- level $\left(x_{i}\right)(=i)$ is the index of $x_{i}$ in $P$, i.e., the distance $d_{G}\left(x_{i}, x_{0}\right)$;

- port $_{u p}\left(x_{i}\right)$ is the port number at vertex $x_{i}$ of the edge $x_{i} x_{i+1}$ (if $i=l$, $\operatorname{port}_{u p}\left(x_{i}\right):=$ nil);

$-\operatorname{port}_{\text {down }}\left(x_{i}\right)$ is the port number at vertex $x_{i}$ of the edge $x_{i} x_{i-1}$ (if $i=0$, port $_{\text {down }}\left(x_{i}\right):=$ nil).

For each vertex $z \in V \backslash P$, we have

$$
\begin{aligned}
\operatorname{Label}(z):= & \left(b(z), \operatorname{level}(z), a_{v}(z), \operatorname{port}_{v-\text { in }}(z), \operatorname{port}_{v-\text { out }}(z), a_{h}(z), \operatorname{port}_{h-\text { in }}(z),\right. \\
& \left.\operatorname{port}_{h-\text { out }}(z), \operatorname{dev}(z), \operatorname{port}_{\text {down }}(z), \operatorname{dev}^{\prime}(z), \operatorname{port}_{u p}(z)\right),
\end{aligned}
$$

where

$-b(z):=0$, a bit indicating that $z$ does not belong to $P$;

- level $(z)$ is the distance $d_{G}\left(z, x_{0}\right)$;

$-a_{v}(z)$ is a bit indicating whether $z$ is adjacent to $x_{l a b e l(z)-1}$;

- $\operatorname{port}_{v-i n}(z)$ is the port number at vertex $x_{\text {label }(z)-1}$ of the edge $x_{\text {label }(z)-1} z$ (if $z$ and $x_{\text {label }(z)-1}$ are not adjacent in $G$, then $\operatorname{port}_{v-i n}(z):=$ nil);

- $\operatorname{port}_{v-\text { out }}(z)$ is the port number at vertex $z$ of the edge $z x_{\text {label(z)-1 }}$ (if $z$ and $x_{\text {label }(z)-1}$ are not adjacent in $G$, then $\operatorname{port}_{v-\text { out }}(z):=$ nil);

- $a_{h}(z)$ is a bit indicating whether $z$ is adjacent to $x_{\text {label }(z)}$;

- $\operatorname{port}_{h-i n}(z)$ is the port number at vertex $x_{\text {label }(z)}$ of the edge $x_{\text {label }(z)} z$ (if $z$ and $x_{\text {label }(z)}$ are not adjacent in $G$, then $\operatorname{port}_{h-i n}(z):=$ nil);

$-\operatorname{port}_{h-o u t}(z)$ is the port number at vertex $z$ of the edge $z x_{\text {label }(z)}$ (if $z$ and $x_{\text {label( } z)}$ are not adjacent in $G$, then $\operatorname{port}_{h-o u t}(z):=$ nil);

$-\operatorname{dev}(z)$ is the deviation of $z$ in tree $T_{2}$;

$-\operatorname{port}_{\text {down }}(z)$ is the port number at vertex $z$ of the edge $z w$, where $w$ is the father of $z$ in $T_{2}$;

- $\operatorname{dev}^{\prime}(z)$ is the deviation of $z$ in tree $T^{\prime}$;

$-\operatorname{port}_{u p}(z)$ is the port number at vertex $z$ of the edge $z w$, where $w$ is the father of $z$ in $T^{\prime}$ (if $\operatorname{dev}^{\prime}(z)=l+1, \operatorname{port}_{u p}(z):=$ nil).

Clearly, the label size of each vertex of $G$ is at most $3\left\lceil\log _{2} l\right\rceil+6\left\lceil\log _{2} \Delta\right\rceil+3 \leq$ $3 \log _{2} \mathcal{D}+6 \log _{2} \Delta+O(1)$ bits.

Routing Decision. The routing decision algorithm is obvious. Suppose that a packet with the header (address of destination) Label $(y)$ arrives at vertex $x$. 
The vertex $x$ can use the following constant time algorithm to decide where to submit the packet. Note that each vertex $v$ of $G$ is uniquely identified by its label Label $(v)$.

\section{function routing_decision_AT-free $(\operatorname{Label}(x), \operatorname{Label}(y))$}

if $\operatorname{Label}(x)=\operatorname{Label}(y)$ then return "packet reached its destination"; else do case

case $(b(x)=1)$

${ }^{*} x$ belongs to $P$ and routing is performed on the caterpillar-tree $T_{1}$ of $G * /$

do case

case $(\operatorname{level}(x)>\operatorname{level}(y))$

send packet via $\operatorname{port}_{\text {down }}(x)$;

case $(\operatorname{level}(x)<\operatorname{level}(y))$

if $b(y)=1$ then send packet via $\operatorname{port}_{u p}(x)$;

else if $\operatorname{level}(y)=\operatorname{level}(x)+1$ and $a_{v}(y)=1$ then

send packet via $\operatorname{port}_{v-i n}(y)$;

else send packet via $\operatorname{port}_{u p}(x)$;

case $(\operatorname{level}(x)=\operatorname{level}(y))$

if $a_{h}(y)=1$ then send packet via $\operatorname{port}_{h-i n}(y)$;

else send packet via port $_{\text {down }}(x)$;

endcase;

/* now $x$ does not belong to $P *$ /

case $(\operatorname{level}(x)>\operatorname{level}(y))$

do case

case $\left(a_{v}(x)=1\right)$

send packet via $\operatorname{port}_{v-\text { out }}(x) ; /$ routing is performed on $T_{1} * /$

case $\left(b(y)=1\right.$ or $b(y)=0$ and $\left.a_{h}(y)=1\right)$

send packet via port $_{h-\text { out }}(x) ; / *$ routing is performed on $T_{1} * /$

otherwise $/ *$ here we have $d_{T_{1}}(x, y)=\operatorname{level}(x)-\operatorname{level}(y)+3 * /$

if $\operatorname{dev}(x) \leq \operatorname{level}(x)-\operatorname{level}(y)+1$ then send packet via $\operatorname{port}_{\text {down }}(x)$;

${ }^{*}$ the cactus-tree $T_{2}$ of $G$ is used for routing */

else send packet via $\operatorname{port}_{h-\text { out }}(x) ; / *$ routing is performed on $T_{1} * /$

endcase;

case $($ level $(x)<\operatorname{level}(y))$

do case

case $\left(a_{h}(x)=1\right)$

send packet via $\operatorname{port}_{h-\text { out }}(x) ; / *$ routing is performed on $T_{1} * /$

case $\left(b(y)=1\right.$ or $b(y)=0$ and $\left.a_{v}(y)=1\right)$

send packet via $\operatorname{port}_{v-\text { out }}(x) ; / *$ routing is performed on $T_{1} * /$

otherwise $/ *$ here we have $d_{T_{1}}(x, y)=\operatorname{level}(y)-\operatorname{level}(x)+3 * /$

if $\operatorname{dev}^{\prime}(x) \leq \operatorname{level}(y)-\operatorname{level}(x)+1$ then send packet via port ${ }_{u p}(x)$;

$/ *$ the willow-tree $T^{\prime}$ of $G$ is used for routing */

else send packet via $\operatorname{port}_{v-\text { out }}(x) ; / *$ routing is performed on $T_{1} * /$ endcase;

case $(\operatorname{level}(x)=\operatorname{level}(y)) / *$ routing is performed on $T_{1} * /$

if $a_{h}(x)=1$ then send packet via $\operatorname{port}_{h-\text { out }}(x)$;

endcase.

else send packet via port $_{v-\text { out }}(x)$;

Thus, we have the following result. 
Theorem 5. Every AT-free graph of diameter $\mathcal{D}:=\operatorname{diam}(G)$ and of maximum vertex degree $\Delta$ admits a $\left(3 \log _{2} \mathcal{D}+6 \log _{2} \Delta+O(1)\right)$-bit routing labeling scheme of deviation at most 2. Moreover, the scheme is computable in linear time, and the routing decision is made in constant time per vertex.

\section{Acknowledgements}

DGC wishes to thank the Natural Sciences and Engineering Research Council of Canada for financial assistance and the Department of Computer Science at Kent State University for hosting his visit there.

\section{References}

1. L.P. CHEw, There are planar graphs almost as good as the complete graph, J. of Computer and System Sciences, 39 (1989), 205-219.

2. D.G. Corneil, S. Olariu and L. Stewart, Asteroidal Triple-free Graphs, SiAM J. Discrete Math., 10 (1997), 399-430.

3. D.G. Corneil, S. Olariu and L. Stewart, Linear time algorithms for dominating pairs in asteroidal triple-free graphs, SIAM J. on Computing, 28 (1999), 1284-1297.

4. F.F. Dragan, C. YAN and I. Lomonosov, Collective tree spanners of graphs, Algorithm Theory - SWAT 2004, 9th Scandinavian Workshop on Algorithm Theory, Humlebaek, Denmark, July 8-10, 2004, Lecture Notes in Computer Science, Vol. 3111, pp. $64-76$.

5. C. Gavoille, M. Katz, N.A. Katz, C. Paul, and D. Peleg, Approximate Distance Labeling Schemes, in Proceedings of the 9th Annual European Symposium on Algorithms" (ESA'01), Aarhus, Denmark, August 28-31, 2001, Lecture Notes in Computer Science 2161, Springer, 2001, pp. 476-487.

6. T. Kloks, D. Kratsch and H. MÜlleR, Approximating the bandwidth for asteroidal triple-free graphs, J. Algorithms, 32 (1999), 41-57.

7. T. Kloks, D. Kratsch and H. MüLler, On the structure of graphs with bounded asteroidal number, Graphs and Combinatorics, 17 (2001), 295-306.

8. A.L. Liestman And T. Shermer, Additive graph spanners, Networks, 23 (1993), 343-364.

9. T.H. MA and J.P. SpInRAD, On the two-chain subgraph cover and related problems, J. of Algorithms, 17 (1994), 251-268.

10. M.S. Madanlal, G. Venkatesan, and C. Pandu Rangan, Tree 3-spanners on interval, permutation and regular bipartite graphs, Inform. Process. Lett., 59 (1996), 97-102.

11. R.M. MCCONNELl and J.P. SpinRAd, Linear-time transitive orientation, In Proceedings of the Eighth Annual ACM-SIAM Symposium on Discrete Algorithms, New Orleans, Louisiana, 5-7 January 1997, pp. 19-25.

12. D. Peleg, Distributed Computing: A Locality-Sensitive Approach, SIAM Monographs on Discrete Math. Appl., SIAM, Philadelphia, 2000.

13. D. Peleg, and A.A. Schäffer, Graph Spanners, J. Graph Theory, 13 (1989), 99-116.

14. D. Peleg And J.D. Ullman, An optimal synchronizer for the hypercube, in Proc. 6th ACM Symposium on Principles of Distributed Computing, Vancouver, 1987, $77-85$.

15. E. Prisner, D. Kratsch, H.-O. Le, H. Müller, and D. Wagner, Additive tree spanners, SIAM Journal on Discrete Mathematics, 17 (2003), 332-340. 